\title{
Clinicopathological features and outcomes of esophageal lesions containing a basal layer type squamous cell carcinoma component
}

This article was published in the following Dove Press journal: Cancer Management and Research

\author{
Xiaoduan Zhuang' \\ Zhenyu Chen' \\ Jianqi Wang ${ }^{2}$ \\ Junsheng Chen' \\ Xiaosheng $\mathrm{Wu}^{\prime}$ \\ Yadong Wang' \\ Chudi Chen' \\ Guoming Deng' \\ Kai Qian' \\ Yang Bai ${ }^{l}$
}

'Department of Gastroenterology, Nanfang Hospital, Southern Medical University, Guangzhou 5105I5, People's Republic of China; ${ }^{2}$ Department of Otolaryngology, The Third Affiliated Hospital of Southern Medical University, Guangzhou 510515, People's Republic of China
Correspondence: Yang Bai

Department of Gastroenterology,

Nanfang Hospital, Southern Medical

University, Guangzhou 5105I5, People's

Republic of China

Tel +86 I 392500 I665

Fax +86 $0206 \quad 164|57|$

Email docbaiyang@sohu.com
Purpose: Basal layer type squamous cell carcinoma (BLSCC) is a unique type of squamous cell carcinoma (SCC), characterized by high-grade dysplastic cells occupying the lower half of the epithelium. So far, such special lesions do not seem to attract much attention. The aim of this study was to investigate the clinicopathological features and prognosis of esophageal squamous carcinoma lesions with a BLSCC component.

Materials and methods: Between January 2011 and January 2018, 96 patients with esophageal squamous cell carcinoma underwent endoscopic submucosal resection in our hospital were retrospectively analyzed. Patients were divided into BLSCC or typical SCC groups according to the presence or absence of a BLSCC component. The endoscopic findings were compared between the two groups. Furthermore, patients were followed up until October 2018 to compare recurrence rates.

Results: BLSCC components were detected in 32 (33.3\%, 32/96) lesions. Among them, 13 $(40.62 \%, 13 / 32)$ were BLSCC predominant. The intraepithelial papillary capillary loops of 7 pure BLSCC showed type B1 under narrow-band imaging. Single-factor and multivariate analyses indicated that five or more independently scattered, deep-stained spots in iodineunstained areas were significantly predictive of the presence of BLSCC components $(\mathrm{OR}=4.837, P=0.015)$. All patients of typical SCC group survived, but one of BLSCC group died for distant metastases during the follow-up period. The 1-year cumulative recurrence rate (CRR) of BLSCC group were 3.4\%, lower than that of typical SCC group (7.1\%). Although no significant difference of CRR was seen between the two groups $(P>0.05)$, the 2-year CRR of BLSCC group increased to $11.9 \%$, being higher than that of typical SCC group (7.1\%).

Conclusion: The presence of multiple, scattered stained spots in iodine-unstained areas was predictive of BLSCC components. Such lesion should be treated actively and subject to a more rigorous follow-up protocol due to a higher likelihood of late recurrence.

Keywords: esophagus, basal layer type squamous cell carcinoma, clinicopathology, recurrence

\section{Introduction}

Basal layer type squamous cell carcinoma (BLSCC) is a unique type of SCC characterized by high-grade dysplastic cells occupying the lower half of the squamous epithelium, ${ }^{1}$ being first proposed by Japanese pathologists in $1999 .^{2}$ As we all know, there are significant differences in the pathological diagnostic criteria used by Western and Japanese pathologists. In Western countries, evident invasion of the lamina propria mucosae (LPM) by dysplastic squamous cells is crucial for diagnosing 
esophageal squamous cell carcinoma (SCC), while Japanese pathologists pay more attention to the individual cellular and architectural changes, particularly, the nuclear findings. ${ }^{3,4}$ This implies that the same lesion diagnosed as dysplasia by Western pathologists may be differently diagnosed as carcinoma in situ or noninvasive carcinoma by Japanese pathologists. BLSCC, which usually be underdiagnosed as low-grade intraepithelial neoplasia (LGIN) according to World Health Organization (WHO) criteria $^{5}$ because of the presence of surface maturation, is an incisive manifestation of the differences in pathological criteria between the East and the West. While, BLSCC is fundamentally different from LGIN that the former has aggressive biological behavior to infiltrate directly into the mucosal lamina propria, which is in contrast to the conventional carcinogenesis pathway of LGIN-HGIN-Invasive SCC. $^{6}$ Based on the theory that esophageal cancer is a common tumor with multicentric occurrences, BLSCC can coexist with typical SCC or true LGIN components, ${ }^{7,8}$ which further increases the difficulties of detecting and correct diagnosis of such lesion. In the past twenty years, few reports concerning BLSCC have been published. In only a few studies, the cases reported by Goda et al, and Zhao et al, showed different endoscopic findings of BLSCC under narrow-band imaging (NBI), as well as lugol chromoendoscopy (LCE). ${ }^{9,10}$ Until now, the clinicopathological characteristics and clinical outcomes of BLSCC are mostly unclear.

This aim of this study is to clarify the clinicopathological characteristics and to investigate the prognosis of 32 cases with a BLSCC component.

\section{Materials and methods}

\section{Patients}

A total of 147 consecutive patients (90 males, 47 females) who underwent endoscopic submucosal resection (ESD) for esophageal squamous epithelial neoplastic lesions, from January 2011 through January 2018, at Nanfang Hospital, Southern Medical University (Guangzhou, China) were identified. Lymph node metastases were not detected in any of these patients on preoperative endoscopic ultrasound or imaging. The exclusion criteria were: 1) history of esophageal-related chemoradiotherapy or endoscopic treatment; 2) did not undergo magnifying endoscopy or iodine staining before ESD; 3) multiple esophageal lesions necessitating two or more ESD surgeries in one day; 4) incomplete clinicopathological data.
The clinicopathological data, including endoscopic findings and pathological reports, of the 96 patients (62 males, 34 females, $\mathrm{M}: \mathrm{F}=31: 17$; age range, 37-86 years; median age, 59.4 years) who were finally included were collected in detail. Written informed consent was taken from all patients after preoperative conversations. The study was conducted in accordance with the ethical standards laid down in the 1964 Declaration of Helsinki and approved by Nanfang Hospital, Southern Medical University ethics committee.

\section{Endoscopic findings}

All the included lesions were first investigated using white light endoscopy (WLE), followed by narrow-band imaging with magnification endoscopy (NBI-ME). The abnormal intrapapillary capillary loops (IPCLs) were classified according to the Japan Esophageal Society classification. ${ }^{11}$ After NBI-ME, the lesion was stained with $1.5 \%$ iodine solution. An iodineunstained area appearing as a worm-eaten boundary was classified as an irregular type (Figure 1A and B), while an area showing a smooth boundary was considered a regular type (Figure 1C and D). As long as there were five or more independently scattered, stained spots in the iodine-unstained area, the area was defined as positive for multiple, scattered, stained spots (Figure 2A), otherwise, the area was identified as negative for multiple, scattered, stained spots (Figure 2B).

\section{Histopathological evaluation}

Hematoxylin-eosin (HE) slides of each of the ESD sections from all patients enrolled in the study were subjected to a 200-fold magnification scan using Aperio CS2, a digital pathology slide scanner (Leica Biosystems Imaging Inc, San Diego, CA, USA). Pathological diagnosis of BLSCC was performed by two separate pathologists according to the 11th edition of the Japanese Classification of Esophageal Cancer $^{1}$, using Aperio ImageScanScope software, version 12.10 (Leica Biosystems). Based on the presence or absence of BLSCC components in the specimens, the 96 patients were divided into a BLSCC group $(n=32)$ and a typical SCC group $(n=64)$. In addition, the immunohistochemical findings of $\mathrm{Ki}-67$ in the BLSCC group were observed.

\section{Follow-up}

Patients were instructed to conduct endoscopic follow-up at 3, 6, 12 months after ESD and every 12 months thereafter. Follow-up data were retrospectively collected from medical records. Incomplete or missing data were retrieved from the referring physicians, patients or their families through 
A

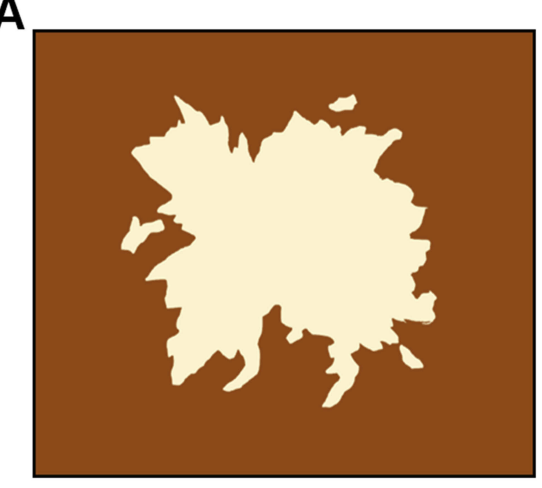

C

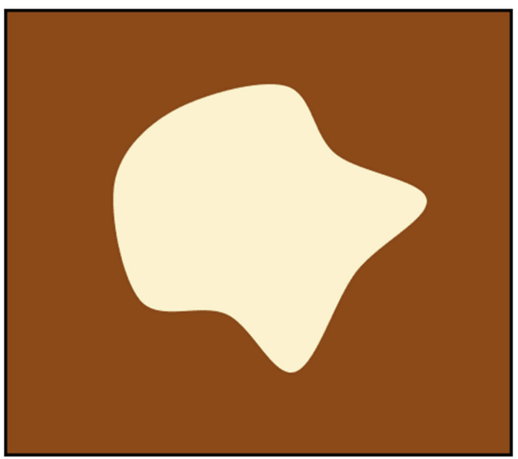

B

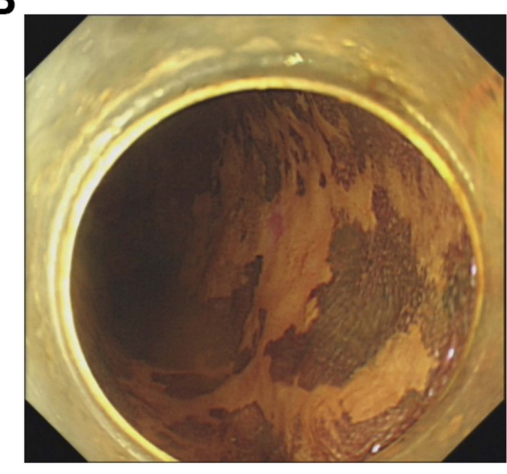

D

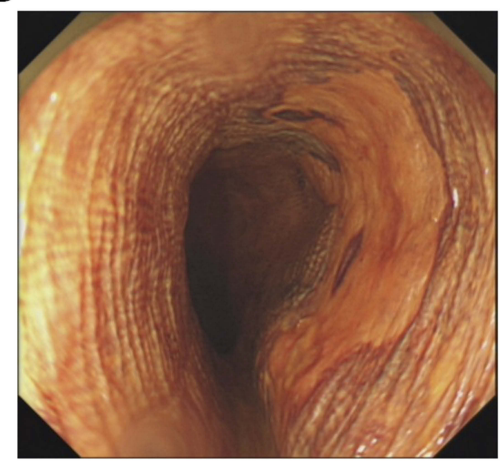

Figure I Images of esophageal squamous epithelial neoplastic lesions after iodine staining. (A) A schematic drawing of the worm-eaten boundary of an iodine-unstained area (irregular type). (B) A lesion with an irregular type of boundary of an iodine-unstained area. (C) A schematic drawing of the smooth boundary of an iodine-unstained area (regular type). (D) A lesion with a regular type and a well-demarcated boundary of an iodine-unstained area.

A

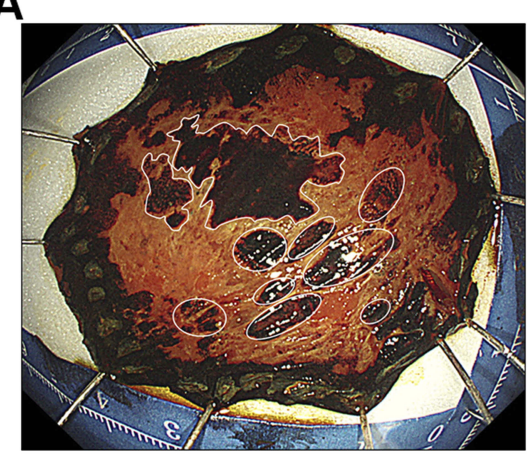

B

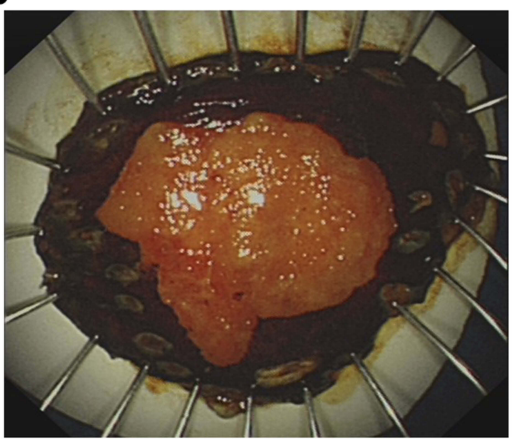

Figure 2 Images of endoscopic submucosal resection specimens stained by Lugol dye solution. (A) A lesion with more than five stained spots (marked by white circles), which were independently scattered in the iodine-unstained area, without connection to the surrounding iodine-stained area, was defined as positive for multiple stained spots in an iodine-free area. (B) A lesion with less than five scattered, deep-stained spots in the iodine-unstained area; negative for multiple stains in the iodine-free area.

telephone conversations. In our study, follow-up was conducted until October 1, 2018. The end point of follow-up for recurrence was defined as the time of recurrence findings under endoscopy, including residual and local recurrence.

\section{Statistical analysis}

IBM SPSS Statistics software (version 22.0, IBM, New

York, NY, USA) was used for statistical analysis of the data. Continuous variables were expressed as mean \pm standard deviation (SD). The clinicopathological data of BLSCC and typical SCC groups were compared using $\chi^{2}$ test, Fisher's exact test, or Student's $t$-test, as appropriate. Multivariate logistic regression analysis was performed to investigate the predictors of the existence of a BLSCC component. Cumulative recurrence rate (CRR) of the two groups was evaluated by the Kaplan-Meier method and 
compared by log-rank test. $P$-value $<0.05$ was considered statistical significance.

\section{Results}

\section{Basic clinicopathologic characteristics}

Of the 96 esophageal squamous epithelial neoplastic lesions, the BLSCC components were detected in 32 (33.3\%, 32/96) lesions. The inter-observer reliability of the diagnosis of BLSCC component in this study was moderate (Kappa $=0.493$ ). The basic clinicopathologic features of the 32 patients are summarized in Table 1. The BLSCC group was composed of 23 males and 9 females, with a median age of 61.3 years (range from 39 to 73 years). All the lesions were located in the middle and lower esophagus, predominantly in the middle esophagus (26, 81.25\%). Histological examination of ESD specimens showed that most of the lesions mixed up with a BLSCC

Table I The clinicopathological features of 32 patients with a BLSCC component

\begin{tabular}{|l|l|}
\hline Characteristics & No. of patients (\%) \\
\hline Sex (male/female) & $23(71.88) / 9(28.13)$ \\
\hline Age (y) & \\
Median & 61.25 \\
Range & $39.0-73.0$ \\
\hline Smoker(male/female) & $15(46.88) / 0(0)$ \\
\hline Alcohol drinker(male/female) & $11(34.38) / 2(6.25)$ \\
\hline Tumor location & \\
Upper esophagus & $0(0)$ \\
Middle esophagus & $26(81.25)$ \\
Lower esophagus & $6(18.75)$ \\
\hline Predominant lesion & \\
BLSCC component & $13(40.63)$ \\
Typical SCC component & $19(59.37)$ \\
\hline Depth of invasion & \\
MI & $15(46.88)$ \\
M2 & $11(34.37)$ \\
M3 & $2(6.25)$ \\
SMI & $1(3.12)$ \\
>SMI & $3(9.38)$ \\
\hline Infiltrative growth pattern & $11(34.38)$ \\
Inf a & 0 \\
Inf b & \\
Inf c & \\
\hline
\end{tabular}

Abbreviations: MI, Intraepithelial carcinoma; M2, tumor invades lamina propria mucosae; M3, tumor invades muscularis mucosae; SMI, tumor invades the upper third of the submucosal layer; Inf, infiltration growth pattern. component and a typical SCC component. Of the 13 $(40.63 \%, 13 / 32)$ lesions with BLSCC as the main component, $7(21.87 \%, 7 / 32)$ were pure BLSCC in situ. All of the 7 lesions performed a biopsy before ESD, but none of the specimens taken were diagnosed as BLSCC but as LGIN.

Microscopically, the BLSCC is characterized by a high degree of architectural and cytological abnormalities confined to the lower half of esophageal epithelium. They presented loss of normal cell polarity, crowded arrangement, marked variation in nuclear size and shape, increase in nuclear/cytoplasmic (N/C) ratio, but existed surface differentiation. There are $21(65.62 \%, 21 / 32)$ lesions showed expansive growth toward LPM, presenting expansive growth of tumor nests with a well-demarcated border from surrounding tissue, like Figure 3A. And 11 (34.38\%) lesions showed incomplete the basal membrane of esophageal epithelium with scatter minimal tumor foci in LPM, like Figure 3B. Immunohistochemical findings of Ki-67 of the BLSCC component suggested that Ki-67 positive cells were mainly concentrated in the lower half of the squamous epithelium, including the basal layer (Figure 3C).

\section{Endoscopic findings}

To investigate the relevant endoscopic features of lesions containing a BLSCC component, we compared endoscopic findings of the BLSCC group $(\mathrm{n}=32,33.3 \%)$ and typical SCC group $(n=64,66.7 \%)$ in Table 2. The two groups did not differ significantly in average age, smoking habits, alcohol consumption, and depth of infiltration of lesions ( $P>0.05$ ), suggesting that the two groups had good comparability. In terms of endoscopic findings, there was no statistical significance in the location of lesions, mucosal surface leukoplakia, macroscopic type between the two groups. Though the two groups showed no difference in IPCL classification $(P=0.449)$, on further analysis of 7 pure BLSCC in situ, we found that the IPCL in the heaviest regions under NBI-ME in all 7 lesions belonged to type B1 (Figure 4). With respect to iodine staining, in the BLSCC group, 29 (90.6\%) lesions had an irregular boundary of iodine-free zone and 27 (84.4\%) lesions had multiple, scattered, stained regions in the iodine-unstained area; both these features were significantly higher in the BLSCC group than in the typical SCC group $(P=0.006$ and $P<0.001$, respectively).

It has been previously reported in the literature that the presence of leukoplakia on the mucosal surface may be a predictor of BLSCC. ${ }^{9}$ Therefore, surface leukoplakia 
A

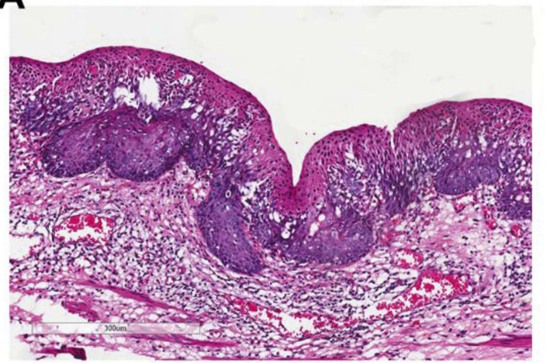

C

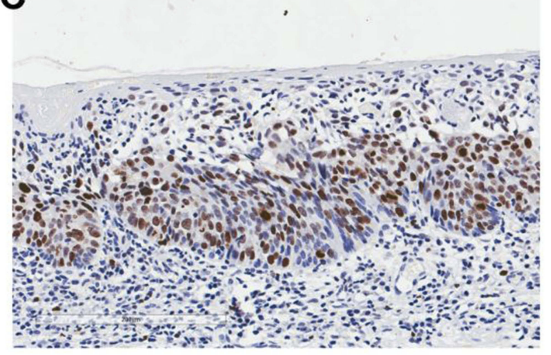

B

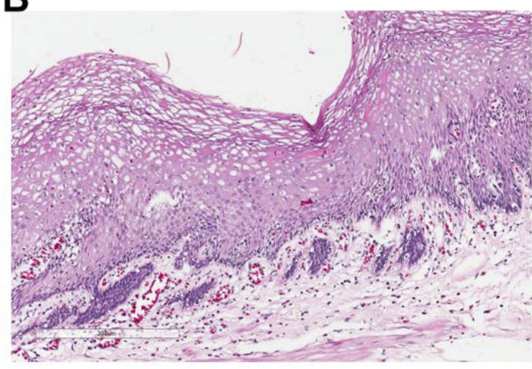

Figure 3 The invasive growth patterns and Ki-67 immunohistological findings of BLSCC components. (A) The BLSCC component presented with an infiltrative growth pattern, INFa, toward the LPM. (B) The BLSCC component exhibited INFb with minimal tumor foci in the LPM. (C) Ki-67 positive cells were mainly detected in the lower half of the epithelial layer of BLSCC components.

Abbreviations: LPM, lamina propria mucosae; INF, infiltration growth pattern.

accompanying an iodine-stained boundary and diffusely stained, small lesions in the non-stained area were included in the multivariate analysis. Presence of multiple, scattered, deep-stained spots in an iodine-unstained area emerged as an independent risk factor for the presence of BLSCC components in esophageal squamous epithelial neoplastic lesions in the analysis ( $\mathrm{OR}=4.837 ; 95 \%$ $\mathrm{CI}=1.357-17.242 ; P=0.015$ ) (Table 3).

\section{Clinical outcomes}

By October 2018, 89 (92.7\%, 89/97) patients were followed up. The median follow-up period is 29.8 months (range: 5.7-63.9 months) in BLSCC group and 27 months (range: $2.5-65.1$ months) in typical SCC group. During the follow-up period, a total of 4 patients in BLSCC group had a local recurrence at 5.7 months, 15.5 months, 23.1 months, and 28.7 months after ESD, respectively. Among the 4 cases, one patient received additional surgery and radiotherapy because of lymph node metastasis but died of tumor recurrence 21.3 months after surgery. The remaining 3 patients underwent the second treatment of ESD. In the typical SCC group, 2 patients were found to have residual at 2.5 and 3.1 months after ESD, respectively. And local recurrence occurred in 2 patients at 11.0 and 11.5 months after ESD, respectively. The above 4 patients were successfully treated with additional ESD. No death was observed in typical SCC group. All the 89 patients underwent at least one lugol chromoendoscopic reexamination during the follow-up period. The 1-year CRR of BLSCC group was $3.4 \%$, slightly lower than that of typical SCC group (7.1\%, Logrank $P=0.507, \quad \mathrm{HR}=1.870, \quad 95 \%$ $\mathrm{CI}=0.294-11.90$ ). While the CRR of BLSCC group was as high as $11.9 \%$ in 2 years, higher than that of typical SCC group $(7.1 \%$, Log rank $P=0.652, \mathrm{HR}=0.699,95 \%$ $\mathrm{CI}=0.147 \sim 3.316$ ). No significant differences of $\mathrm{CRR}$ were seen between the two groups (Figure 5).

\section{Discussion}

BLSCC is a distinct variant of esophageal SCC proposed by Japanese pathologists. So far, few references have been concerning BLSCC, most of them from Asian studies. ${ }^{7,9,10}$ The possible geographical aspects may be related to the relatively low incidence of esophageal squamous cell carcinoma in the west. In addition, different criteria for pathological diagnosis of carcinoma between the east and the west are also one of the reasons that cannot be ignored. Because of its deceptive appearance that the dysplasia cells are confined to the lower half of squamous epithelium, it usually is misdiagnosed as LGIN by pathologists who follow WHO criteria in the pathological diagnosis process, sequentially suffering from the risk of missing opportunities for timely treatment. ${ }^{7}$ The fact that none of 
Table 2 Comparison between BLSCC and typical SCC groups, n (\%)

\begin{tabular}{|c|c|c|c|c|}
\hline Variables & & BLSCC $(n=32)$ & Typical SCC $(n=64)$ & $P$-value \\
\hline Ages (year, mean $\pm S D$ ) & & $61.25 \pm 7.94$ & $58.45 \pm 9.20$ & 0.146 \\
\hline Gender (n) & Male/female & $23 / 9$ & $39 / 25$ & 0.291 \\
\hline Smoker & & $15(46.9)$ & $21(32.8)$ & 0.180 \\
\hline Alcohol drinker & & $13(40.6)$ & $16(25.0)$ & 0.116 \\
\hline Tumor location & $\begin{array}{l}\text { Upper third } \\
\text { Middle third } \\
\text { Lower third }\end{array}$ & $\begin{array}{l}0 \\
26(81.3) \\
6(19.4)\end{array}$ & $\begin{array}{l}2(3.1) \\
53(82.8) \\
9(13.8)\end{array}$ & $0.698^{*}$ \\
\hline IPCL types & $\begin{array}{l}\text { B1 } \\
\text { B2 } \\
\text { B3 }\end{array}$ & $\begin{array}{l}24(75) \\
6(18.8) \\
2(6.3)\end{array}$ & $\begin{array}{l}4 I(64 . I) \\
20(3 I .3) \\
3(4.7)\end{array}$ & $0.449 *$ \\
\hline BC & $\begin{array}{l}\text { Positive } \\
\text { Negative }\end{array}$ & $\begin{array}{l}26(81.3) \\
6(18.8)\end{array}$ & $\begin{array}{l}59(92.2) \\
5(7.8)\end{array}$ & $0.172^{*}$ \\
\hline Leukoplakia & $\begin{array}{l}\text { Positive } \\
\text { Negative }\end{array}$ & $\begin{array}{l}\mid 4(43.8) \\
\mid 8(56.3)\end{array}$ & $\begin{array}{l}16(25) \\
48(75)\end{array}$ & 0.062 \\
\hline Boundary of iodine staining & $\begin{array}{l}\text { Regular } \\
\text { Irregular }\end{array}$ & $\begin{array}{l}3(9.4) \\
29(90.6)\end{array}$ & $\begin{array}{l}23(35.9) \\
4 I(64.1)\end{array}$ & 0.006 \\
\hline Multiple stains in iodine-free area & $\begin{array}{l}\text { Yes } \\
\text { No }\end{array}$ & $\begin{array}{l}27(84.4) \\
5(15.6)\end{array}$ & $\begin{array}{l}28(43.8) \\
36(56.2)\end{array}$ & $<0.001$ \\
\hline Macroscopic type & $\begin{array}{l}\text { Ila } \\
\text { Ilb } \\
\text { Ilc }^{a}\end{array}$ & $\begin{array}{l}7(21.9) \\
17(53.1) \\
8(25.0)\end{array}$ & $\begin{array}{l}12(18.8) \\
38(59.4) \\
14(21.9)\end{array}$ & 0.843 \\
\hline Depth of invasion & $\begin{array}{l}\text { LGIN } \\
\text { HGIN-LPM } \\
\text { MM-SMI } \\
>\text { SMI }\end{array}$ & $\begin{array}{l}0 \\
24(75) \\
5(15.6) \\
3(9.4)\end{array}$ & $\begin{array}{l}8(12.5) \\
38(59.4) \\
15(23.4) \\
3(4.7)\end{array}$ & $0.085^{*}$ \\
\hline $\mathrm{DI}$ or $\mathrm{Gl}$ & $\begin{array}{l}\text { Positive } \\
\text { Negative }\end{array}$ & $\begin{array}{l}12(37.5) \\
20(62.5)\end{array}$ & $\begin{array}{l}20(31.3) \\
44(68.7)\end{array}$ & 0.540 \\
\hline
\end{tabular}

Notes: a Containing Ilc and mixed llc types. *Fisher's exact test.

Abbreviations: BC, background coloration; LPM, tumor invades lamina propria mucosae; MM, tumor invades muscularis mucosae; SMI, tumor invades the upper third of the submucosal layer; DI, ductal involvement; GI, glandular involvement.

the 7 pure BLSCC lesions in our study were correctly diagnosed as BLSCC but as LGIN based on histological specimens taken by endoscopic biopsy, also proved the phenomenon.

Actually, more than a decade ago, Shimizu et al, had presented a special lesion, wherein LGIN was associated with invasion into the LPM. ${ }^{12}$ According to them, an early invasion is not always associated with HGIN and can develop directly from LGIN. Besides, Syafriadi et al, proposed an interesting concept of the two-phase appearance of oral epithelial dysplasia, which was characterized by a sharp change in cell feature between the lower and upper layers, highly similar to BLSCC in histology. ${ }^{13}$
Subsequently, the study by Kobayashi et al, confirmed that in the two-phase epithelial dysplasia lesions, Ki-67 positive cells were aggregated in the lower half including the basal layer. ${ }^{14}$ While in LGIN lesions, Ki-67 positive cells were mainly located in the parabasal rather than basal layer. In addition, the study by Takeda et al, found that the Ki-67 labeling index of the basal layer in two-phase epithelial dysplasia showed no significant difference between that in severe dysplasia and carcinoma in situ. ${ }^{15}$ Based on the above, it suggested that the two-phase epithelial dysplasia was related to malignancy and belonged to high-grade dysplasia group. Similarly, in our study, the Ki67 positive cells were mainly presented in the lower half 
A

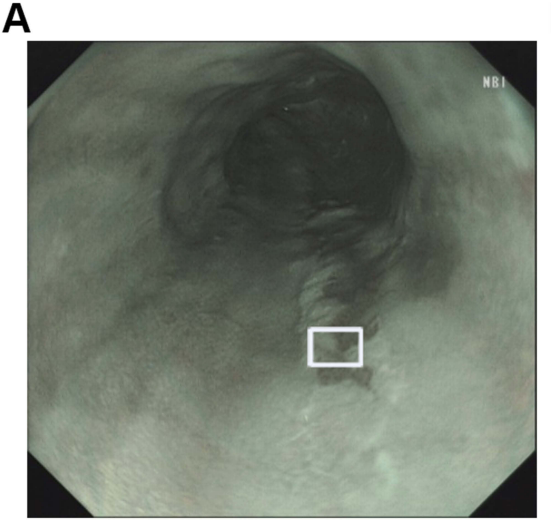

B

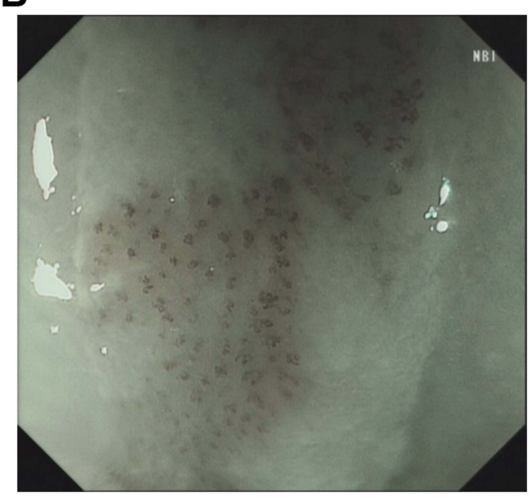

Figure 4 The NBI images of BLSCC. (A) Endoscopic image under NBI showed the lesion with brownish color change. (B) A magnified view of the white box area in Figure A. The IPCLs exhibited the changes of dilatation, meandering, irregular caliber, and form variation, belonging to JES type BI.

Abbreviations: NBI, narrow-band imaging; IPCL, intraepithelial papillary capillary loop; JES, Japan Esophageal Society.

Table 3 Multivariate analysis of variables associated with the presence of BLSCC components

\begin{tabular}{|l|l|l|l|}
\hline Variables & Statue & OR & 95\% Cl \\
\hline Surface leukoplakia & $\begin{array}{l}\text { No } \\
\text { Yes }\end{array}$ & I & $(0.656,4.513)$ \\
\hline Boundary of iodine staining & Regular & I.720 & \\
\hline Multiple stains in iodine-free area & Irregular & I & $(0.366,8.635)$ \\
\hline
\end{tabular}

Abbreviations: $\mathrm{Cl}$, confidence interval; $\mathrm{OR}$, odds ratio.

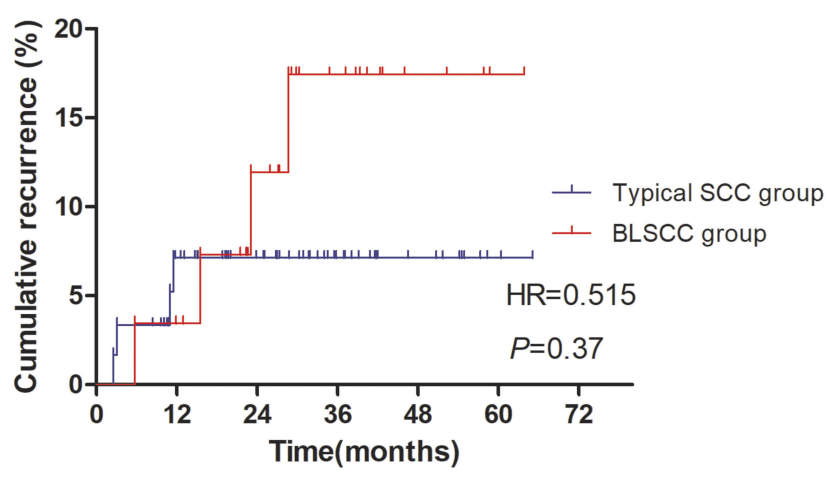

Figure 5 Comparison of cumulative recurrence rates between BLSCC group and typical SCC group.

of BLSCC, showing a higher potential of malignant proliferation than that in LGIN. However, this kind of special lesions does not seem to have attracted much attention. This may be largely attributed to the uncertain definition of BLSCC. Based on previous reports as well as the findings in our study, BLSCC usually coexists with typical SCC components or true LGIN components, ${ }^{7,10}$ and it is still undetermined how to consider the proportion of BLSCC component when making a pathological diagnosis. Considering that a lesion was formed by a combination of small lesions that arose a multicentric occurrence of SCC or dysplasia, ${ }^{7}$ any lesion containing a BLSCC component is bound to be biologically different from typical SCC, regardless of the proportion of BLSCC component. Therefore, in our study, we classified all the 32 lesions with a BLSCC component into BLSCC group. Among them, $13(40.62 \%, 13 / 32)$ case were BLSCC predominant. The pure BLSCC accounted for only $21.87 \%$ (7/32) in our study. Most of the patients with a BLSCC component were male. All of the 32 lesions were located at the middle and lower esophagus. After a detailed evaluation of the ESD specimens, we summarized the histological characteristics of the BLSCC components as follows: 1) high degree of cellular atypia confined to the lower half of squamous epithelium; 2) surface differentiation; 3) loss of normal cell polarity; 4) irregular extension or minimal invasion into the LPM in some cases; 5) enlargement and hyperchromasia of cell nuclei, increased N/C ratio; 6) Ki-67 positive cells were mainly present in the subepithelial $1 / 2$ 
layer, including the basal layer. These histological findings are largely supported by the previous reports of Zhao et al, and Goda et $\mathrm{al}^{9,10}$. The biological behavior of early infiltration into LPM suggests that BLSCC are more aggressive and essentially different from LGIN. The early detection and diagnosis of this type of lesions have a significant impact on the prognosis of patients.

NBI-ME is an effective means of detecting esophageal lesions and predicting their depth. ${ }^{16}$ Goda et al, reported that in a lesion consisting of both histological components, namely BLSCC and true LGIN, the IPCL density in the epithelial region corresponding to LGIN was low, showing only mild morphological changes. However, in the region where BLSCC was located, IPCL showed high density, accompanied by significant morphological changes: expansion, distortion, different tube diameters, and different morphology. ${ }^{10}$ In a further analysis of 7 pure BLSCC lesions, we found that the IPCL in the heaviest regions under NBI-ME in all lesions showed marked morphological changes, belonging to type $\mathrm{B} 1$, a finding similar to that of Goda et al. We believe this finding is an important hint for clinically reducing the misdiagnose rate of BLSCC. In cases where LGIN is diagnostically proven on the biopsy, but the IPCL morphology is classified as type B1, it needs to further confirm whether there existed a BLSCC component.

Lugol chromoendoscopy is another traditional and effective means to detect esophageal dysplasia. However, to date, research about the endoscopy performances of BLSCC has been rather scarce. A case report by Zhao et al, described BLSCC as exhibiting asymmetrical leukoplakia under WLE, and the boundaries of the iodineunstained areas as being clear. ${ }^{9}$ Similarly, our study found that the probability of leukoplakia on the surface of the lesion in BLSCC group was higher than that in typical SCC group, although the difference was not statistically significant $(43.8 \%$ vs $25 \%, P=0.062)$. However, contrary to the results of iodine staining observed by Zhao et al, we found that the iodine-unstained regions exhibited a significant boundary irregularity in BLSCC group compared to those in typical SCC group $(90.6 \%$ vs $60.1 \%, P=0.006$ ). In addition, we found that the iodineunstained areas of BLSCC group were more likely to have multiple, scattered, deep-stained small foci than that of typical SCC group $(84.4 \%$ vs $43.8 \%, \quad P<0.001)$. Furthermore, multivariate analysis showed that multiple, scattered, deep-stained small foci of the iodine-unstained areas were an independent risk factor (relative risk, 4.837; $P=0.015)$ for the presence of BLSCC components. To our knowledge, akin findings have never been reported before. Previous studies have been confirmed that glycogen granules were found almost not in the basal layer but in the superficial layer of nonkeratinized epithelium. Subsequently, iodine is distributed mainly in the upper third of the superficial layer of esophageal epithelium after iodine staining. ${ }^{17,18}$ Since the superficial layer above BLSCC is near normal, glycogen still remains in the residual normal epithelium to react to iodine. Thus, we speculate that the diffuse, deep-stained, small foci in the iodine-unstained areas most likely correspond to the scattered distribution of BLSCC components in the pathological tissues. Prospective studies are expected to test this hypothesis in the future. But on the basis of what we found, we suggested that lesions with multiple (more than 5), scattered, deep-stained small foci in iodineunstained areas should be suspected of the existence of BLSCC components and taken seriously.

BLSCC exhibits a similar cytological appearance to an invasive squamous cell carcinoma, ${ }^{19}$ which indicates laterally that such lesions have the same or higher invasiveness than typical SCC. So far, the standard treatment for BLSCC has not been established due to the difficulties of diagnosis. In this study, ESD was performed for the BLSCC group, which was the same as that for typical SCC group. During the 29.8 months of median follow-up periods, one patient from BLSCC group died of tumor recurrence. In addition, the lesions with BLSCC components seem to present a higher recurrence trend. The 2year cumulative recurrence rate of BLSCC group was higher than those of typical SCC group (11.9\% vs 7.1\%). But no statistical differences of cumulative recurrence rates were seen between the two groups, which may be due to the insufficient follow-up time or lack of a large number of cases. It needs to be investigated by more welldesigned prospective studies. The recurrence time of all 4 cases in our study from typical SCC group was within 1 year after ESD, which is almost in line with the previous reports. $^{20,21}$ However, the recurrence time of BLSCC group seems to be later than that of typical SCC group. Of the 4 recurrent cases, the time between ESD and recurrence in 3 cases $(75 \%, 3 / 4)$ was more than 1 year. Therefore, based on our experience, BLSCC should be actively treated in early stage. Furthermore, different from the traditional follow-up protocol for typical SCC, ${ }^{22}$ BLSCC should be closely followed up even one year after ESD treatment. 
So far, the possible molecular or pathological basis of BLSCC is still unclear. According to the research by Syafriadi et al, they discovered that the characteristic twophase appearance of oral epithelial dysplasia was associated with the apoptotic phenomena of prickle cells, mostly resulted from the intraepithelial infiltrations of both cytotoxic T cells and natural killer cell. ${ }^{13}$ In view of the fact that the BLSCC is highly similar to the two-phase appearance, it is reasonable to assume that the apoptosis of spinous cells may be a possible mechanism behind BLSCC. Further research is expected to test this hypothesis.

There are some limits in our study. This study was a retrospective study with a small sample size of basal layer type SCC predominant lesions. A multicenter prospective study is required. Further research about a specific molecular marker that can distinguish the clinicopathological difference between typical SCC and BLSCC is expected to provide an efficient and objective basis for correct diagnosis.

In general, our study is the first report focused on the clinicopathologic feature and prognosis of lesions with a BLSCC component based on the largest case series. We demonstrated that BLSCC was an aggressive malignant tumor, which is difficult to be diagnosed via biopsy. The presence of multiple, scattered, deep-stained small foci in an iodine-unstained area of a lesion, especially one presenting with type B1 IPCL under NBI-ME, should alert an endoscopic physician to the possibility of a BLSCC component. Such lesions seem to have a later recurrence than typical SCC, thus requiring a more rigorous follow-up protocol.

\section{Acknowledgment}

The authors would like to thank International Science Editing for editing this manuscript.

\section{Author contributions}

All authors contributed to data analysis, drafting or revising the article, gave final approval of the version to be published, and agree to be accountable for all aspects of the work.

\section{Disclosure}

The authors report no conflicts of interest in this work.

\section{References}

1. Japan Esophageal Society. Japanese classification of esophageal cancer, 11th edition: part II and III. Esophagus-Tokyo. 2017;14(1):37-65. doi:10.1007/s10388-016-0556-2.
2. Japanese Society of Esophageal Diseases. [Guidelines for the Clinical and Pathologic Studies on Carcinoma of the Esophagus] 9th edn. Tokyo: Kanehara, 1999. (In Japanese.).

3. Schlemper RJ, Kato Y, Stolte M. Diagnostic criteria for gastrointestinal carcinomas in Japan and Western countries: proposal for a new classification system of gastrointestinal epithelial neoplasia. $J$ Gastroenterol Hepatol. 2010;15(s3):G49-G57. doi:10.1046/j.14401746.2000.02266.x

4. Schlemper RJ, Kato Y, Stolte M. Review of histological classifications of gastrointestinal epithelial neoplasia: differences in diagnosis of early carcinomas between Japanese and Western pathologists. $J$ Gastroenterol. 2001;36(7):445-456. doi:10.1007/s005350170067

5. Shimizu M, Nagata K, Yamaguchi H, Kita H. Squamous intraepithelial neoplasia of the esophagus: past, present, and future. $J$ Gastroenterol. 2009;44(2):103-112. doi:10.1007/s00535-008-2298-y

6. Ohashi S, Miyamoto SI, Kikuchi O, et al. Recent advances from basic and clinical studies of esophageal squamous cell carcinoma. Gastroenterology. 2015;149(7):1700-1715. doi:10.1053/j.gastro.2015.08.054

7. Shimizu Y, Yoshida T, Kato M, et al. Low-grade dysplasia component in early invasive squamous cell carcinoma of the esophagus. $J$ Gastroen Hepatol. 2010;25(2):314-318. doi:10.1111/j.14401746.2009.06032.x

8. Kuwano H, Saeki H, Kawaguchi H, et al. Proliferative activity of cancer cells in front and center areas of carcinoma in situ and invasive sites of esophageal squamous-cell carcinoma. Int J Cancer. 1998;78 (2):149-152. doi:10.1002/(SICI)1097-0215(19981005)78:2<149:: AID-IJC4>3.0.CO;2-Z

9. Zhao Y, Fu YW, Sun Q. A unique lesion of the esophageal mucosal epithelium: low-grade intraepithelial neoplasia or basal-layer-type squamous cell carcinoma? Chin Med J (Engl). 2017;130(13):16191620. doi:10.4103/0366-6999.208230

10. Goda K, Tajiri H, Ikegami M, Dobashi A, Yoshimura N. Clinical impact of narrow-band imaging magnifying endoscopy for 'basal layer type squamous cell carcinoma' in the esophagus. Dig Endosc. 2011;23:75-78. doi:10.1111/j.1443-1661.2011.01121.x

11. Oyama T, Inoue H, Arima M, et al. Prediction of the invasion depth of superficial squamous cell carcinoma based on microvessel morphology: magnifying endoscopic classification of the Japan Esophageal Society. Esophagus-Tokyo. 2017;14(2):105-112. doi:10.1007/s10388-016-0527-7

12. Shimizu M, Ban S, Odze RD, Squamous dysplasia and other precursor lesions related to esophageal squamous cell carcinoma. Gastroenterol Clin North Am. 2007;36(4):797-811. doi:10.1016/j. gtc. 2007.08 .005

13. Syafriadi M, Cheng J, Jen KY, et al. Two-phase appearance of oral epithelial dysplasia resulting from focal proliferation of parabasal cells and apoptosis of prickle cells. J Oral Pathol Med. 2005;34 (3):140-149. doi:10.1111/j.1600-0714.2004.00283.x

14. Kobayashi T, Maruyama S, Cheng J, et al. Histopathological varieties of oral carcinoma in situ: diagnosis aided by immunohistochemistry dealing with the second basal cell layer as the proliferating center of oral mucosal epithelia. Pathol Int. 2010;60(3):156-166. doi:10.1111/ j.1440-1827.2009.02499.x

15. Takeda T, Sugihara K, Hirayama Y, et al. Immunohistological evaluation of Ki-67, p63, CK19 and p53 expression in oral epithelial dysplasias. J Oral Pathol Med. 2010;35(6):369-375. doi:10.1111/ j.1600-0714.2006.00444.x

16. Goda K, Tajiri H, Ikegami M, et al. Magnifying endoscopy with narrow band imaging for predicting the invasion depth of superficial esophageal squamous cell carcinoma. Dis Esophagus. 2009;22 (5):453-460. doi:10.1111/j.1442-2050.2009.00942.x

17. Aizawa H, Yamada S, Xiao T, et al. Difference in glycogen metabolism (glycogen synthesis and glycolysis) between normal and dysplastic/malignant oral epithelium. Arch Oral Biol. 2017;83:340347. doi:10.1016/j.archoralbio.2017.08.014 
18. Xiao T, Kurita $\mathrm{H}$, Li X, et al. Iodine penetration and glycogen distribution in vital staining of oral mucosa with iodine solution. Oral Surg Oral Med Oral Pathol Oral Radiol. 2014;117(6):754759. doi:10.1016/j.oooo.2014.02.008

19. Takubo K. Pathology of esophagus. In: An Atlas and Textbook. 2nd ed. Vol. 100. Tokyo: Springer; 2007. p. 145-190.

20. Esaki M, Matsumoto T, Hirakawa K, et al. Risk factors for local recurrence of superficial esophageal cancer after treatment by endoscopic mucosal resection. Endoscopy. 2007;39(1):41-45. doi:10.1055/s-2006-945143
21. Kim DH, Jung HY, Gong EJ, et al. Endoscopic and oncologic outcomes of endoscopic resection for superficial esophageal neoplasm. Gut Liver. 2015;9(4):470-477. doi:10.5009/gnl13263

22. Kitagawa Y, Uno T, Oyama T, et al. Esophageal cancer practice guidelines 2017 edited by the Japan esophageal society: part 2 . Esophagus-Tokyo. 2019;16(1):25-43. doi:10.1007/s10388-018$0642-8$

\section{Publish your work in this journal}

Cancer Management and Research is an international, peer-reviewed open access journal focusing on cancer research and the optimal use of preventative and integrated treatment interventions to achieve improved outcomes, enhanced survival and quality of life for the cancer patient.
The manuscript management system is completely online and includes a very quick and fair peer-review system, which is all easy to use. Visit http://www.dovepress.com/testimonials.php to read real quotes from published authors. 